\title{
Closed-form approximations of the peak-to-average power ratio distribution for multi-carrier modulation and their applications
}

\author{
Marwa Chafii ${ }^{*}$, Jacques Palicot ${ }^{1}$ and Rémi Gribonval ${ }^{2}$
}

\begin{abstract}
The theoretical analysis of the peak-to-average power ratio (PAPR) distribution for an orthogonal frequency division multiplexing (OFDM) system, depends on the particular waveform considered in the modulation system. In this paper, we generalize this analysis by considering the generalized waveforms for multi-carrier (GWMC) modulation system based on any family of modulation functions, and we derive a general approximate expression for the cumulative distribution function (CDF) of its PAPR, for both finite and infinite integration time. These equations allow us to directly find the expressions of the PAPR distribution for any particular functions and characterize the behaviour of the PAPR distribution associated with different transmission and observation scenarios. In addition to that, a new approach to formulating the PAPR reduction problem as an optimization problem, is presented in this study.
\end{abstract}

Keywords: Distribution; Peak-to-average power ratio (PAPR); Generalized waveforms for multi-carrier (GWMC); Orthogonal frequency division multiplexing (OFDM); Multi-carrier modulation (MCM); Optimization

\section{Introduction}

Orthogonal frequency division multiplexing (OFDM) [1-3] is a technique used to send information over several orthogonal carriers in a parallel manner. Compared to the single-carrier modulation, this system shows a better behaviour with respect to frequency-selective channels and gives a better interference reduction. It allows an optimal use of the bandwidth, thanks to the orthogonality of its carriers (compared to FDMA systems). These are among the reasons why this technique has been adopted in many standards such as the asymmetric digital subscriber line (ADSL) [4], the Digital Audio Broadcasting (DAB) [5], the Digital Video Broadcasting-Terrestrial (DVB-T2) [6], HiperLAN/2 [7], Long Term Evolution (LTE) and WiMAX.

However, the OFDM signal features large amplitude variations compared to that of the single carrier's signal, because it is the sum of many narrowband signals in the time domain, with different amplitudes. Based on this fact, in-band and out-of-band distortions occur during the

*Correspondence: marwa.chafii@supelec.fr

1 SUPELEC/IETR, Cesson-Sévigné Cedex 35576, France

Full list of author information is available at the end of the article introduction of the signal into a non-linear device, such as the high-power amplifier (HPA). In order to study these high amplitude fluctuations, the peak-to-average power ratio (PAPR) has been defined. The PAPR is a random variable, as the symbols arrive randomly at the modulation input. To study this measure, several researchers have analysed the distribution law for different particular modulation bases: the Fourier basis [8], the wavelet basis [9], the wavelet packet basis [10] and the discrete cosine transform (DCT) [11]. Others have studied the PAPR based on the Fourier modulation basis, using different waveforms, such as the square root raised cosine (SRRC) [12], the isotropic orthogonal transform algorithm (IOTA) [13], the extended Gaussian functions (EGF) and other prototype filters in the PHYDYAS project [14] for the filter bank multi-carrier (FBMC). For the wavelet modulation basis, we can also study the PAPR by using several types of wavelets, such as the Daubechies wavelets, the Haar wavelet and the biorthogonal wavelet [15]. To the best of our knowledge, this is the first work that generalizes the previously done studies, by considering any family of modulation functions, so as to derive an approximation of the PAPR distribution. The equations are applied to check the expressions of the PAPR distribution, which have been 
derived by other authors for the conventional OFDM and FBMC. In addition, the PAPR distribution, which is based on the general expression, is validated by means of a simulation for the non-orthogonal FDM (NOFDM) $[16,17]$ and for the Walsh-Hadamard multi-carrier (WH-MC) [18]. This work also shows how the PAPR reduction problem can be formulated as a constrained optimization problem, which opens new research perspectives for the scientific community.

We first describe the generalized waveforms for multicarrier (GWMC) system considered in our derivations in Section 2 and derive its PAPR distribution in Section 3. In Section 4, some applications of the PAPR approximation are explained. Finally, Section 5 concludes the paper.

\section{System description}

We consider in this section the most general theoretical case: transmitting infinite data in a continuous way over time. We first model the GWMC signal at the modulation output in Section 2.1. In Section 2.2, we define the conditions that our system must satisfy for the analysis of the PAPR distribution in Section 3.

\subsection{GWMC system}

Information-bearing symbols, resulting from modulation performed by any type of constellation, are decomposed into several blocks. Each block of symbols is inserted in parallel into a modulation system as shown in Figure 1. At the output of the modulator, the GWMC signal can be expressed as [19]:

\section{Definition 1. (GWMC signal)}

$$
X(t)=\sum_{n \in Z} \sum_{m=0}^{M-1} C_{m, n} \underbrace{g_{m}(t-n T)}_{g_{m, n}(t)} .
$$

- $T$, duration of a block of input symbols

- $M$, number of carriers that we assume greater than 8 . (This is an assumption made for the validity of central limit theorem (CLT); for more details, please refer to Berry-Essen's theorem in Appendix 1

- $C_{m, n}=C_{m, n}^{R}+j C_{m, n}^{I}$, input symbols from a mapping technique that take complex values

- $\left(g_{m}\right)_{m \in \llbracket 0, M-1 \rrbracket}, \in L^{2}(\mathrm{R})$ (the space of square integrable functions), family of functions representing the modulation system with $g_{m, n}(t)=g_{m, n}^{R}(t)+j g_{m, n}^{I}(t)$

- $X(t)=X^{R}(t)+j X^{I}(t)$

\subsection{Assumptions}

In order to apply the generalized central limit theorem (G-CLT) in Section 3, our system must satisfy the following two assumptions:

Assumption 1. (Independence of input symbols)

- $\left(C_{m, n}^{R}\right)_{(m \in \llbracket 0, M-1 \rrbracket, n \in \mathrm{Z})}$ are pairwise independent, each with zero mean and a variance of $\frac{\sigma_{c}^{2}}{2}$.

- $\left(C_{m, n}^{I}\right)_{(m \in \llbracket 0, M-1 \rrbracket, n \in \mathrm{Z})}$ are also pairwise independent, each with zero mean and a variance of $\frac{\sigma_{c}^{2}}{2}$.

- $(\forall m, p \in \llbracket 0, M-1 \rrbracket),(\forall n, q \in z) C_{m, n}^{R}$ and $C_{p, q}^{I}$ are independent.

As a consequence,

$$
\begin{aligned}
& E\left(C_{m, n}^{R} C_{p, q}^{R}\right)=\frac{\sigma_{c}^{2}}{2} \delta_{m, p} \delta_{n, q}, \\
& E\left(C_{m, n}^{I} C_{p, q}^{I}\right)=\frac{\sigma_{c}^{2}}{2} \delta_{m, p} \delta_{n, q}, \\
& E\left(C_{m, n}^{R}\right)=E\left(C_{m, n}^{I}\right)=0, \\
& E\left(C_{m, n}^{R} C_{p, q}^{I}\right)=E\left(C_{m, n}^{R}\right) E\left(C_{p, q}^{I}\right)=0 .
\end{aligned}
$$

The symbols from constellation diagrams of the usual digital modulation schemes (quadrature amplitude modulation (QAM), quadrature phase shift keying (QPSK), etc.) satisfy these conditions.
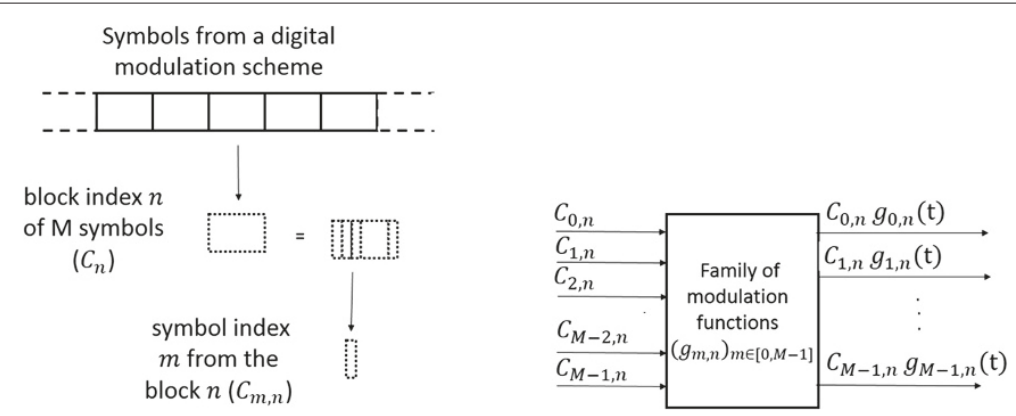

Figure 1 GWMC system. 
Assumption 2.

$$
\begin{aligned}
& \max _{m, t} \sum_{n \in Z}\left|g_{m}(t-n T)\right|=B_{1}<+\infty \\
& \text { and } \min _{m, t} \sum_{n \in Z}\left|g_{m}(t-n T)\right|^{2}=A_{2}>0 .
\end{aligned}
$$

As discussed in Appendix 2B, Assumption 2 is sufficient to ensure Lyapunov's conditions, which ensures the validity of a variant of the CLT that considers independent random variables which are not necessarily identically distributed [20]. The reader can refer to Appendices 1 and 2A for more details.

\section{An analysis of the PAPR}

\subsection{General definition of the continuous-time PAPR}

For a finite observation duration of $N T$, we can define the PAPR for the GWMC signal expressed in Equation 1 as follows:

$$
\operatorname{PAPR}_{c}^{N}=\frac{\max _{t \in[0, N T]}|X(t)|^{2}}{P_{c, \text { mean }}} .
$$

The index $c$ corresponds to the continuous-time context and the exponent $N$ is the number of GWMC's symbols considered in our observation.

Lemma 1. (Mean power of GWMC signal)

$$
\begin{aligned}
P_{c, \text { mean }} & =\lim _{t_{0} \rightarrow+\infty} \frac{1}{2 t_{0}} \int_{-t_{0}}^{t_{0}} E\left(|X(t)|^{2}\right) d t \\
& =\frac{\sigma_{c}^{2}}{T} \sum_{m=0}^{M-1}\left\|g_{m}\right\|^{2} .
\end{aligned}
$$

The mean power $P_{c \text {,mean }}$ is defined over an infinite integration time, because our scenario assumes an infinite transmission time. The details of its derivation, in order to obtain Equation 9, are explained in Appendix 3.

For the discrete-time context, the PAPR can be defined as follows:

Definition 2. (Discrete-time PAPR of GWMC signal for a finite observation duration)

$$
\begin{aligned}
& P_{A P R_{d}^{N}}=\frac{\max _{k \in \llbracket 0, N P-1 \rrbracket}|X(k)|^{2}}{P_{d, \text { mean }}} \\
& P_{d, \text { mean }}=\frac{\sigma_{c}^{2}}{P} \sum_{m=0}^{M-1}\left\|g_{m}\right\|^{2}, \\
& \text { such that } \quad\left\|g_{m}\right\|^{2}=\sum_{k=-\infty}^{+\infty}\left|g_{m}(k)\right|^{2} .
\end{aligned}
$$

The subscript $d$ corresponds to the discrete-time context, and $P$ is the number of samples in period $T$.
In fact, the discrete mean power is defined as

$$
P_{d \text {,mean }}=\lim _{K \rightarrow+\infty} \frac{1}{2 K+1} \sum_{k=-K}^{K} E\left(|X(k)|^{2}\right) .
$$

Siclet has derived in his thesis [21] the mean power of a discrete BFDM/QAM (biorthogonal frequency division multiplexing) signal that is expressed as $X[k]=\sum_{m=0}^{M-1} \sum_{n=-\infty}^{+\infty} f_{m}[k-n P]$, such that $f_{m}[k]$ is an analysis filter. His derivation does not use the exponential property of $f_{m}[k]$. Then, in our case, we can follow the same method to get Equation 11.

To simplify the derivation of an approximation of the PAPR distribution, we will consider the discrete-time definition of the PAPR.

\subsection{Approximation of the CDF of the PAPR}

The CDF or its complementary function is usually used in the literature as a performance criterion of the PAPR. The CDF is the probability that a real-valued random variable (the PAPR here) featuring a given probability distribution will be found at a value less than or equal to $\gamma$, which can be expressed in our case as

$$
\operatorname{Pr}\left(\mathrm{PAPR}_{d}^{N} \leq \gamma\right)=\operatorname{Pr}\left[\frac{\max _{k \in \llbracket 0, N P-1 \rrbracket}|X(k)|^{2}}{P_{d, \text { mean }}} \leq \gamma\right]
$$

Lemma 2. (Upper bound of the PAPR)

For a finite number of carriers $M$, and a family of functions $\left(g_{m}\right)_{m \in \llbracket 0, M-1 \rrbracket}$ that satisfies $A_{2}=\min _{m, t}$ $\sum_{n \in Z}\left|g_{m}(t-n T)\right|^{2}>0$ and $B_{1}=\max _{m, t}$ $\sum_{n \in Z}\left|g_{m}(t-n T)\right|<+\infty$, we have for any observation duration I, and any input symbols,

$$
\begin{aligned}
& \operatorname{PAPR}_{c}(I) \leq C M:=P A P R_{c, \text { bound }}, \\
& \text { with } C=\frac{\max _{m, n}\left|C_{m, n}\right|^{2} B_{1}^{2}}{\sigma_{C}^{2} A_{2}} .
\end{aligned}
$$

Note that $\operatorname{PAPR}_{c, \text { sup }}=\operatorname{supPAPR}_{I}(I) \leq \mathrm{PAPR}_{c \text {,bound }}$. For proof and a full demonstration, please refer to Appendix 4.

Based on Lemma 2, there is a finite PAPR for any $\gamma>\mathrm{PAPR}_{c \text {,sup }}$ and any observation duration $I$, $\operatorname{Pr}\left(\operatorname{PAPR}_{c}(I) \leq \gamma\right)=1$, the CCDF is then equal to zero beyond the $\mathrm{PAPR}_{c \text {,sup }}$ (see Figure 2). We can also mention that the bound goes to infinity when the number of carriers $M$ goes to infinity, the CCDF is then equal to 1 for any sufficiently small $\gamma$ (see Figure 2).

In what follows, we give an approximation of the CDF of the PAPR for $\gamma$ sufficiently small compared with

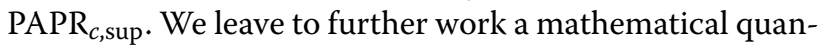
tification of how small $\gamma$ should be. For finite $N$, the experiments show a good fit with our prediction. 


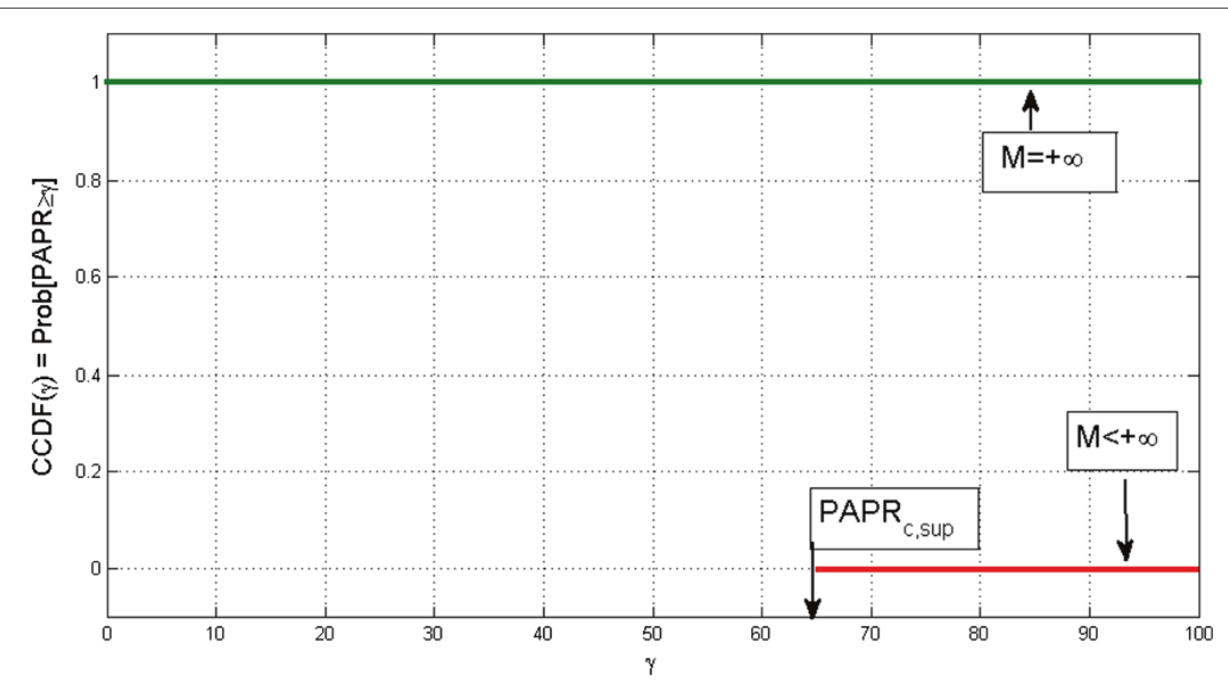

Figure 2 PAPR distribution beyond its supremum.

By considering an observation duration limited to $N$ GWMC symbols of $P$ samples each, and by approximating the samples $X(0), X(1), X(2), \ldots, X(N P-1)$ as being independent (which is the approximation made in [8] in the absence of oversampling), the CDF of the PAPR for the GWMC signal can be expressed as follows:

$$
\begin{aligned}
\operatorname{Pr}\left(\operatorname{PAPR}_{d}^{N} \leq \gamma\right) & =\operatorname{Pr}\left[\max _{k \in \llbracket 0, N P-1 \rrbracket}|X(k)|^{2} \leq \gamma P_{d, \text { mean }}\right] \\
& =\operatorname{Pr}\left(\forall k \in \llbracket 0, N P-1 \rrbracket|X(k)|^{2} \leq \gamma P_{d, \text { mean }}\right) \\
& \approx \prod_{k \in \llbracket 0, N P-1 \rrbracket} \operatorname{Pr}\left(|X(k)|^{2} \leq \gamma P_{d, \text { mean }}\right) .
\end{aligned}
$$

We should now look for the distribution law of $|X(k)|^{2}$. We first find the distribution of the real part of $X(k)$ and, after that, do the same for the imaginary part.

Let us put

$$
\begin{aligned}
X_{m}^{R}(k) & =\sum_{n \in \mathrm{Z}} C_{m, n}^{R} g_{m, n}^{R}(k)-C_{m, n}^{I} g_{m, n}^{I}(k) \\
X_{m}^{I}(k) & =\sum_{n \in Z} C_{m, n}^{R} g_{m, n}^{I}(k)+C_{m, n}^{I} g_{m, n}^{R}(k), \\
\text { such that } \quad X^{R}(k) & =\sum_{m=0}^{M-1} X_{m}^{R}(k) \\
X^{I}(k) & =\sum_{m=0}^{M-1} X_{m}^{I}(k) .
\end{aligned}
$$

We have the random variables $X_{0}^{R}(k), X_{1}^{R}(k), X_{2}^{R}(k), \ldots$, $X_{M-1}^{R}(k)$ that are independent with zero mean and satisfy Lyapunov's condition by assuming Assumption 2. Thus, for large $M$, we can apply the G-CLT (see Appendix 2) and get

$$
\begin{aligned}
& \sum_{m=0}^{M-1} X_{m}^{R}(k) \sim \mathcal{N}(\underbrace{0, \frac{\sigma_{c}^{2}}{2} \sum_{n \in Z} \sum_{m=0}^{M-1}\left|g_{m, n}(k)\right|^{2}}_{\frac{\sigma_{X}^{2}(k)}{2}}) \\
& X^{R}(k) \sim \mathcal{N}\left(0, \frac{\sigma_{X}^{2}(k)}{2}\right) .
\end{aligned}
$$

Following the same steps, we get

$$
X^{I}(k) \sim \mathcal{N}\left(0, \frac{\sigma_{X}^{2}(k)}{2}\right)
$$

Then $X(k)$ follows a complex Gaussian process with zero mean and variance $\sigma_{X}^{2}(k)=\sigma_{c}^{2} \sum_{n \in Z} \sum_{m=0}^{M-1}$ $\left|g_{m}(k-n P)\right|^{2}$. Hence,

$$
|X(k)|^{2} \sim \chi^{2} \text { with two degrees of freedom. }
$$$$
\text { Denoting } \quad x(k)=\frac{P_{d \text {, mean }}}{\sigma_{X}^{2}(k)} \text {, }
$$

we obtain from Equations 16 and 20 the following result:

Theorem 1. (PAPR distribution of GWMC signal for a finite observation duration)

For large $M$, with the considered simplifying assumptions, we have

$$
\begin{aligned}
& \operatorname{Pr}\left(\operatorname{PAPR}_{d}^{N} \leq \gamma\right) \approx \prod_{k \in \llbracket 0, N P-1 \rrbracket}\left[1-\mathrm{e}^{-x(k) \gamma}\right], \\
& \text { with } \quad x(k)=\frac{\sum_{m=0}^{M-1}\left\|g_{m}\right\|^{2}}{P \sum_{n \in Z} \sum_{m=0}^{M-1}\left|g_{m}(k-n P)\right|^{2}} .
\end{aligned}
$$


Note that the approximate distribution of the PAPR depends not only on the family of modulation functions $\left(g_{m}\right)_{m \in \llbracket 0, M-1 \rrbracket}$ but also on the number of carriers $M$ used. In addition, it depends on the parameter $N$, the number of GWMC symbols observed. Taking into account the simplifying assumption of our derivations, we can easily study the PAPR performance of any multi-carrier modulation (MCM) system. The approximate expression of the CDF of the PAPR will be compared to the empirical CDF featured in Section 4.1. In Section 3.3, we deduce the behaviour of the PAPR distribution for an infinite observation period.

\subsection{PAPR distribution for an infinite observation period}

By considering an infinite number of GWMC symbols observed, we can define the PAPR as being

$$
\operatorname{Pr}\left(\mathrm{PAPR}_{d}^{\infty} \leq \gamma\right)=\operatorname{Pr}\left[\frac{\max _{k \in \mathrm{N}}|X(k)|^{2}}{P_{d, \text { mean }}} \leq \gamma\right] .
$$

To get the PAPR distribution in this case, we can let $N$ go to infinity in Equation 21; then, we obtain the following formula:

Corollary 1. (PAPR distribution of GWMC signal for an infinite observation duration)

For large $M$, with the considered simplifying assumptions, we have

$$
\operatorname{Pr}\left(\operatorname{PAPR}_{d}^{\infty} \leq \gamma\right)=0
$$

In fact, we have

$$
\begin{aligned}
\operatorname{Pr}\left(\mathrm{PAPR}_{d}^{\infty} \leq \gamma\right) & =\prod_{k \in \mathrm{N}}\left[1-\mathrm{e}^{-x(k) \gamma}\right] \\
\text { with } \quad x(k) & =\frac{\sum_{m=0}^{M-1}\left\|g_{m}\right\|^{2}}{P \sum_{n \in \mathrm{Z}} \sum_{m=0}^{M-1}\left|g_{m}(k-n P)\right|^{2}}
\end{aligned}
$$

$$
\begin{aligned}
& \text { And we have } \sum_{m=0}^{M-1}\left\|g_{m}\right\|^{2}=\sum_{m=0}^{M-1} \sum_{n \in Z} \sum_{k=0}^{P-1}\left|g_{m}(k-n P)\right|^{2} \text {, } \\
& \text { then } \quad A_{2} M P<\sum_{m=0}^{M-1}\left\|g_{m}\right\|^{2}<B_{2} M P
\end{aligned}
$$

$$
\text { and } \frac{1}{B_{2} M P}<\frac{1}{M \sum_{n \in \mathrm{Z}} \sum_{m=0}^{M-1}\left|g_{m, n}(k)\right|^{2}}<\frac{1}{A_{2} M P}
$$

From Equations 25 and 26, we get

$$
\frac{A_{2}}{B_{2}}<x(k)<\frac{B_{2}}{A_{2}}
$$

hence

$$
\left(1-\mathrm{e}^{-x(k) \gamma}\right)<1-\mathrm{e}^{-\frac{B_{2}}{A_{2}} \gamma}<1
$$

Thus, for an infinite observation duration, and for $\gamma$ sufficiently small compared with $\mathrm{PAPR}_{c \text {,sup, we have }}$

$$
\operatorname{Pr}\left(\operatorname{PAPR}_{d}^{\infty} \leq \gamma\right)=0
$$

The CCDF is then equal to 1 for any $\gamma$ (see Figure 3 ).

Equation 29 can be interpreted by the fact that we cannot control the PAPR for an infinite number of GWMC symbols, because we will inevitably have some GWMC symbols with large peaks. Thus, for $M \geq 8$ and $\gamma$ sufficiently small compared with $\mathrm{PAPR}_{c \text {,sup }}$ and an infinite observation duration, there is no family of modulation functions $\left(g_{m}\right)_{m \in \llbracket 0, M-1 \rrbracket}$ that can avoid the large peaks. Based on this fact, we expect to optimize the PAPR within a finite number of GWMC symbols. As the number of GWMC symbols decreases, PAPR optimization gets better.

\section{Applications}

The approximation of the PAPR distribution law for any modulation system provides a valuable contribution to the study of the PAPR problem in the case of MCM systems. In particular, it allows us a fast computation of the PAPR distribution for any family of modulation functions that satisfies our conditions. Based on developed equations, we are able to characterize the behaviour of the PAPR distribution for different transmission and observation scenarios and for any interval of $\gamma$. In addition to that, we show how the PAPR reduction problem can be formulated as an optimization problem. We give more details in the following sections.

\subsection{Simple computation of the PAPR for any family of modulation functions}

We know that the PAPR is a random variable, that is why the derivation of its distribution law may be complex. Especially if you have to repeat the derivation each time the family of modulation functions changes. Equation 21 is a closed form approximation, so it allows us to directly find an approximate CDF of the PAPR without complex operations. To illustrate this, we consider the following examples.

\subsubsection{Conventional OFDM}

We want to check the expression derived by Van Nee in [8] for the conventional OFDM for the discrete case. Then, let us consider the following: 


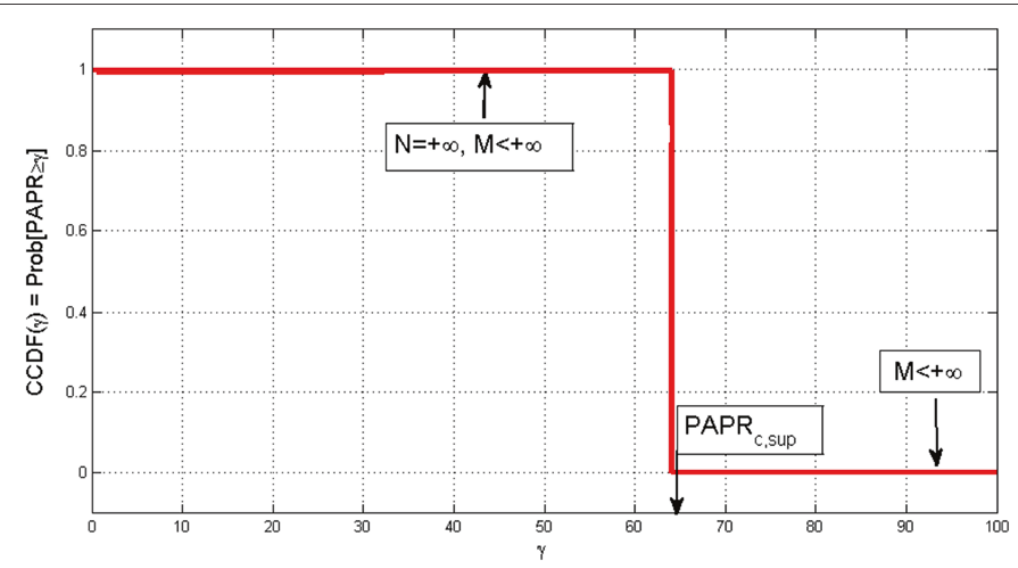

Figure 3 CCDF of the PAPR for an infinite observation duration.

- The Fourier basis is used for the modulation, with a rectangular waveform:

$$
\begin{array}{r}
g_{m}(k)=\mathrm{e}^{\frac{j 2 \pi m k}{P}} \Pi_{[0, P]}(k) \\
\text { with } \Pi_{[0, P]}(k)=\left\{\begin{array}{ll}
1 & \text { if } 0 \leq k \leq P \\
0 & \text { else }
\end{array} .\right.
\end{array}
$$

- The observation is limited to one OFDM symbol, and the number of samples considered is $M$ then $P=M$.

The expression of the GWMC signal in Equation 1 becomes $X(k)=\sum_{n \in \mathrm{Z}} \sum_{m=0}^{M-1} C_{m, n} \mathrm{e}^{\frac{j 2 \pi m(k-n P)}{P}} \Pi_{[0, P]}(k-n P)$.

We apply Equation 21 of the PAPR distribution with $N=1$. We then get

$$
x(k)=\frac{M P}{P \sum_{n \in \mathrm{Z}} \sum_{m=0}^{M-1}\left|\mathrm{e}^{\frac{j 2 \pi m(k-n P)}{P}} \Pi_{[0, P]}(k-n P)\right|^{2}}=1,
$$

and then

$$
\operatorname{Pr}\left(\mathrm{PAPR}_{d}^{1} \leq \gamma\right)=\left[1-\mathrm{e}^{-\gamma}\right]^{M} .
$$

It is similar to the expression derived by Van Nee for the discrete case.

\subsubsection{FBMC systems}

We consider the OFDM/OQAM as being an example of the FBMC system, and we check the expression derived by Skrzypczak [22]. Then, let us consider the following:

- The modulation functions are

$$
\begin{aligned}
g_{m}(k-n P)= & h_{\mathrm{OQAM}}(k-n P) \\
& \mathrm{e}^{j 2 \pi \frac{m}{M}}(k-D / 2) \mathrm{e}^{j \theta_{m, n}},
\end{aligned}
$$$$
\text { then } \quad\left|g_{m}(k-n P)\right|^{2}=h_{\mathrm{OQAM}}^{2}(k-n P) \text {, }
$$

where $h_{\mathrm{OQAM}}$ is the prototype filter (IOTA, SRRC, PHYDYAS...).

- The observation is limited to one block of symbols, and the number of samples considered in this block is $M$, then $P=M$.

\section{- $\left\|g_{m}\right\|^{2}=1$.}

The expression of the GWMC signal in Equation 1 becomes $X(k)=\sum_{n \in Z} \sum_{m=0}^{M-1} C_{m, n} h_{\mathrm{OQAM}}$ $(k-n P) \mathrm{e}^{j 2 \pi \frac{m}{M}}(k-D / 2) \mathrm{e}^{j \theta_{m, n}}$.

We apply Eq. (21) of the discrete-time PAPR for a finite observation duration with $N=1$. Then, we get

$$
x(k)=\frac{1}{M \sum_{n \in \mathrm{Z}} h_{\mathrm{OQAM}}^{2}(k-n P)}
$$

and then

$$
\operatorname{Pr}\left(\mathrm{PAPR}_{d}^{1} \leq \gamma\right)=\prod_{k=0}^{M-1}\left[1-\mathrm{e}^{\frac{-\gamma}{M \sum_{n \in \mathrm{Z}} h_{\mathrm{OQAM}}^{2}(k-n P)}}\right]
$$

This result is similar to the one obtained by Skrzypczak.

\subsubsection{NOFDM systems}

The family of modulation functions used in NOFDM systems is not orthogonal, but it forms an incomplete Riesz basis. The choice of the non-orthogonal pulses is flexible and can be adapted to the knowledge of the channel; therefore, NOFDM systems have a better bandwidth efficiency than OFDM systems and tends to be more robust against frequency-selective fading [16]. To provide an example of an NOFDM system, we consider Hamming window $w(t)$, which is a non-orthogonal pulse. Thus, the waveform of the modulation is expressed as

$$
\begin{aligned}
g_{m}(t) & =\mathrm{e}^{\frac{j 2 \pi m t}{T}} w(t), \\
w(t) & =\left\{\begin{array}{l}
0.54-0.46 \cos \left(2 \pi \frac{t}{T}\right) \text { if } 0 \leq t \leq T \\
0 \quad \text { else }
\end{array}\right.
\end{aligned}
$$

Figure 4 shows the theoretical CCDF of the PAPR based on Equation 1 with $P=M$ and $N=1$ and 


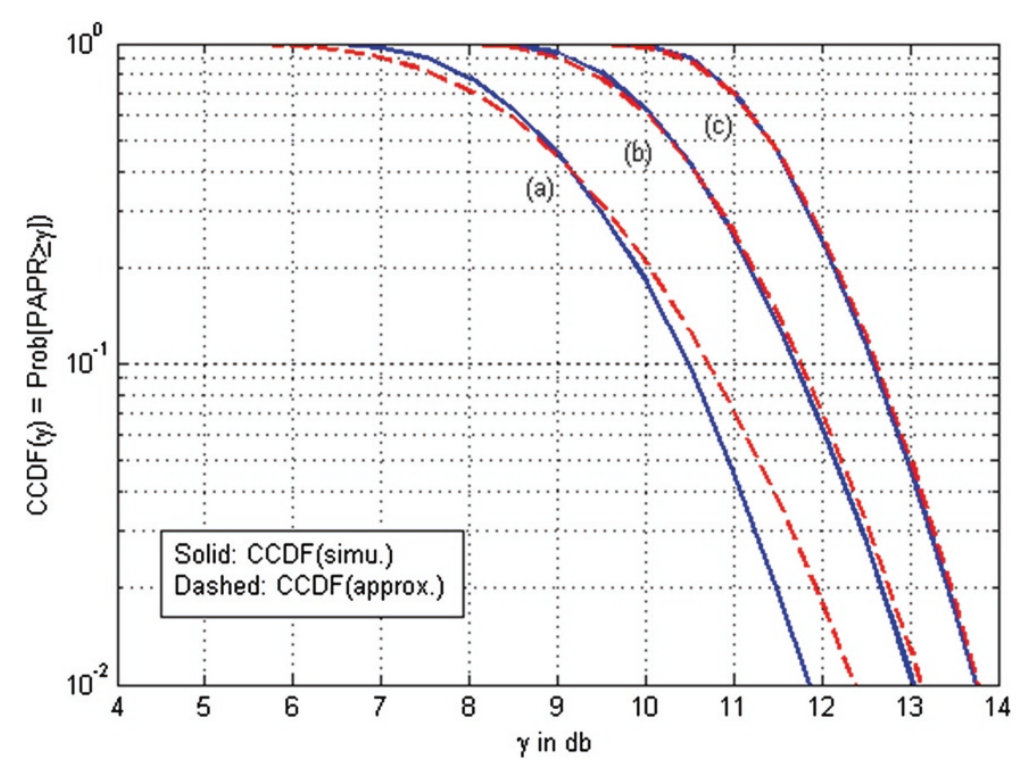

Figure 4 Experimental and theoretical CCDF of PAPR for NOFDM system using Hamming window for different number of carriers. (a) $M=64$, (b) $M=256$ and (c) $M=1,024$.

the experimental CCDF simulated by generating 10,000 realizations of NOFDM symbols, for different number of carriers.

We observe that the larger the number of carriers gets, the more accurate the theoretical curve gets. And this is due to the fact that our derivations are based on the G-CLT which assumes a large number of carriers.

\subsubsection{WH-MC systems}

Instead of using the inverse discrete Fourier transform (IDFT) for the modulation, we can use the inverse WalshHadamard transform (IWHT). Then, the family of the modulation functions is expressed as

$$
g_{m}(k)=W A L_{m}(k)
$$

$\left(W A L_{m}\right)_{m \in \llbracket 0, M-1 \rrbracket}$ are the Walsh functions and are columns of Hadamard matrix of dimension $M=2^{Q}$, which is defined by the recursive formula:

$$
\begin{aligned}
& H\left(2^{1}\right)=\left(\begin{array}{cc}
1 & 1 \\
1 & -1
\end{array}\right) \\
& H\left(2^{2}\right)=\left(\begin{array}{cccc}
1 & 1 & 1 & 1 \\
1 & -1 & 1 & -1 \\
1 & 1 & -1 & -1 \\
1 & -1 & -1 & 1
\end{array}\right),
\end{aligned}
$$

and for $2 \leq q \leq Q$,

$$
H\left(2^{q}\right)=\left(\begin{array}{l}
H\left(2^{q-1}\right) H\left(2^{q-1}\right) \\
H\left(2^{q-1}\right) H\left(2^{q-1}\right)
\end{array}\right)=H(2) \otimes H\left(2^{q-1}\right),
$$

where $\otimes$ denotes the Kronecker product.
Note that the Hadamard matrix consists only of +1 and -1 entries, that is why the implementation has a simple structure featuring only additions and subtractions. In fact, the IWHT can be implemented using the radix2 algorithm, which means that there are only $M \log _{2} M$ complex additions required [23].

Figure 5 shows the theoretical CCDF of the PAPR based on Equation 21 with $P=M$ and $N=1$, and the experimental CCDF simulated by generating 10,000 realizations of WH-MC symbols, for different number of carriers.

We observe that we have a good approximation for $M \geq 64$.

\subsection{CCDF of the PAPR for different transmission and observation scenarios}

The CCDF of the PAPR depends on the number of carriers $M$, the observation duration $N T$ and the parameter $\gamma$ to which the PAPR is compared. For an infinite number of carriers, the PAPR is always large and the CCDF is then equal to 1 for any $\gamma$. If the number of carriers $M$ is finite, the CCDF of the PAPR is equal to 0 for $\gamma$ greater than $\mathrm{PAPR}_{c, \text { sup }}$. And for $\gamma$ less than $\mathrm{PAPR}_{c, \text { sup }}$, the CCDF depends on the observation duration: when we consider an infinite observation duration, the CCDF is equal to 1 , but if we limit the observation duration to a finite number of symbols, the CCDF is defined by Equation 21. Figure 6 presents all the possible cases.

\subsection{Optimization problem}

To reduce the occurrence probability of the peaks, several techniques can be used [24-26]. These techniques take 


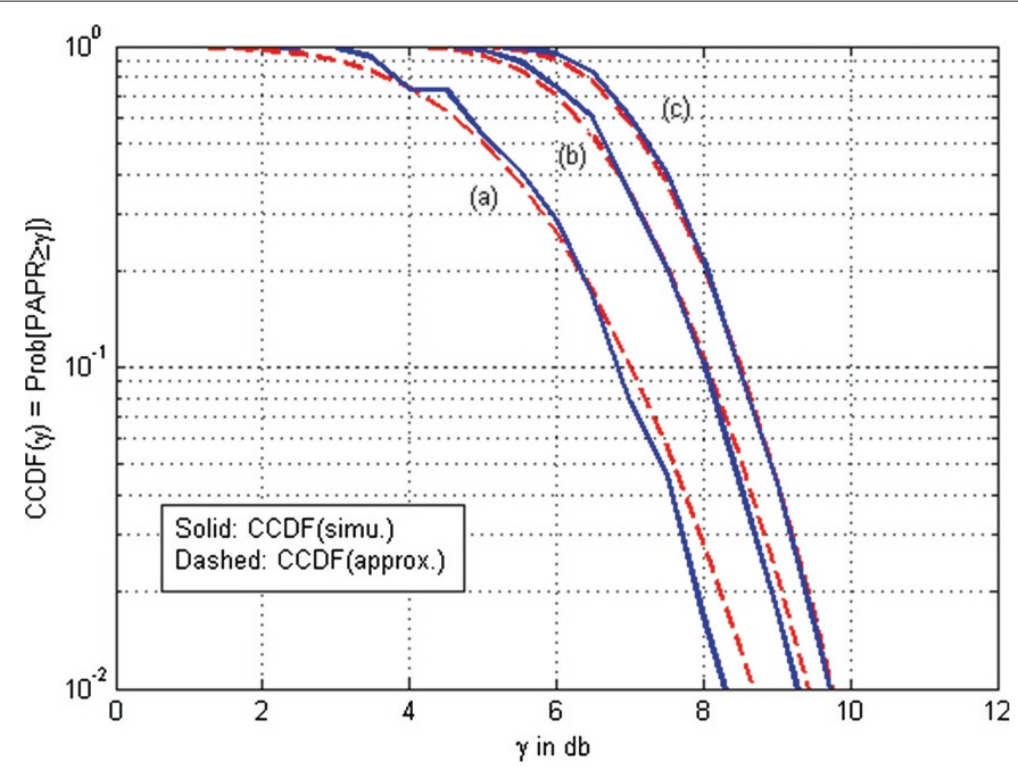

Figure 5 Experimental and theoretical CCDF values of PAPR for a WH-MC system for different number of carriers. (a) $M=64$, (b) $M=256$ and (c) $M=1,024$.

place before and after the modulation. But as we can see in Equation 21, the distribution of the PAPR depends on the family of modulation functions used in the system. Thus, we can act also on the modulation system to reduce the PAPR. In fact, to confirm this idea, we can compare the PAPR performance of the OFDM/OQAM [13] to that of the wavelet OFDM [27]. The first variant uses the same modulation basis as the conventional OFDM (Fourier basis) and does not show any improvement on the PAPR's performance [22], unlike the second that uses the wavelet basis and shows an improvement of up to $2 \mathrm{~dB}[28]$.

Let us model the PAPR's optimization problem. It consists in finding the optimal functions $g_{m}$ that maximize the CDF of the PAPR in Equation 21. By trying to do that, we notice that we should do the optimization at some particular points of the functions $g_{m}$ (the sampling points), while these points are not chosen in a particular way.

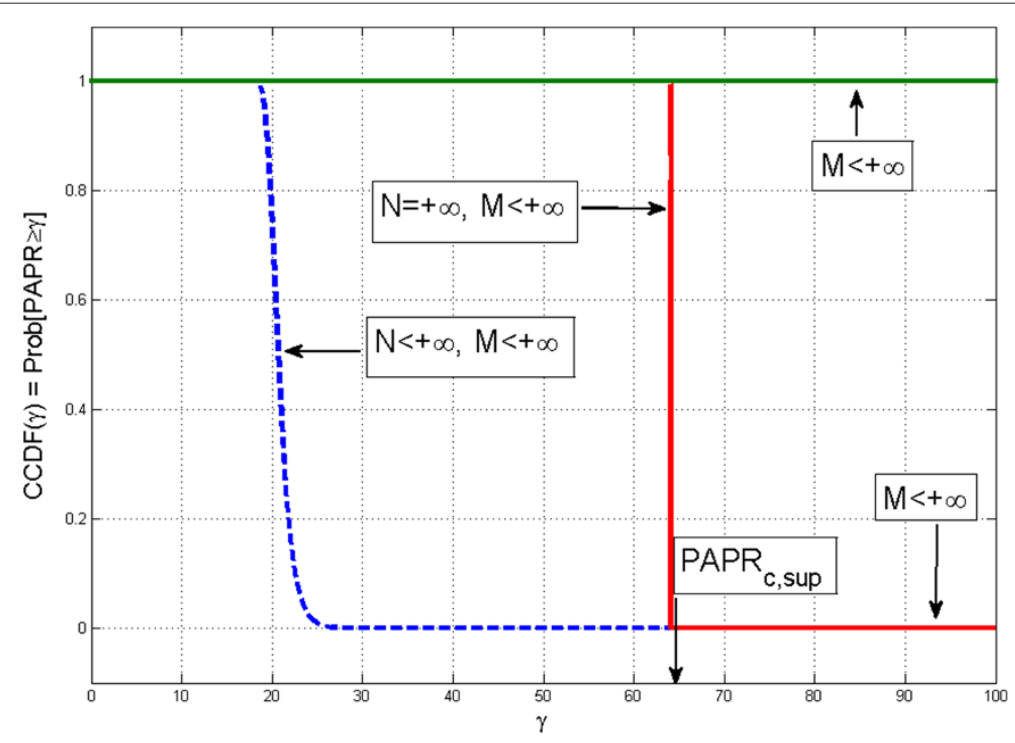

Figure 6 PAPR distribution for different transmission and observation scenarios. 
To adjust the optimization problem, we approximate the denominator of $x(k)$. Let us put

$$
\begin{aligned}
S_{p} & =\ln \left(\operatorname{Pr}\left(\operatorname{PAPR}_{d}^{N} \leq \gamma\right)\right) \\
& =\sum_{k=0}^{N P-1} \ln \left(1-\mathrm{e}^{-\frac{\sum_{m=0}^{M-1}\left\|g_{m}\right\|^{2}}{P \sum_{n \in \mathrm{Z}} \sum_{m=0}^{M-1}\left|g_{m, n}\left(k_{P}^{T}\right)\right|^{2}}} \gamma\right),
\end{aligned}
$$

and we put

$$
h(x)=\ln \left(1-\mathrm{e}^{-\frac{\sum_{m=0}^{M-1}\left\|g_{m}\right\|^{2}}{P \sum_{n \in \mathrm{Z}} \sum_{m=0}^{M-1}\left|g_{m, n}(x)\right|^{2}} \gamma}\right),
$$

We have $h:[0, N T] \rightarrow \mathrm{R}$ is piecewise continuous over $[0, N T]$, and we have

$$
\begin{aligned}
\frac{N T}{N P} S_{p}= & \frac{N T}{N P} \sum_{k=0}^{N P-1} h\left(k \frac{N T}{N P}\right) \quad \text { is a Riemann sum, then } \\
\frac{T}{P} S_{p} & \approx \int_{0}^{N T} h(x) d x \\
\Rightarrow \quad \frac{1}{P} S_{p} & \approx \frac{1}{T} \int_{0}^{N T} h(x) d x \\
\Rightarrow \quad S_{p} & =\frac{P}{T} \int_{0}^{N T} h(x) d x+o(P) \\
\Rightarrow \quad \operatorname{Pr}\left(\operatorname{PAPR}_{d}^{N} \leq \gamma\right) & =\mathrm{e}^{\frac{P}{T} \int_{0}^{N T} h(x) d x}+o(P) .
\end{aligned}
$$

Maximizing $\operatorname{Pr}\left(\operatorname{PAPR}_{d}^{N} \leq \gamma\right)$ is equivalent to maximizing $\int_{0}^{N T} h(x) d x$, and it is equivalent to maximizing $\int_{0}^{T} h(x) d x$ since $h$ is a periodic function of $T$. Then, the quantity that we should maximize over the functions $\left(g_{m}\right)_{m \in \llbracket 0, M-1 \rrbracket}$ is

$$
\int_{0}^{T} \ln \left(1-\mathrm{e}^{-\frac{\sum_{m=0}^{M-1}\left\|g_{m}\right\|^{2}}{P \sum_{n \in \mathrm{Z}} \sum_{m=0}^{M-1}\left|g_{m, n}(t)\right|^{2}} \gamma}\right) d t .
$$

To express the optimization problem that can lead to a new modulation system featuring an optimally reduced PAPR, we have to express the constraints on the functions $\left(g_{m}\right)_{m \in \llbracket 0, M-1 \rrbracket}$. Let us define the following constraints:

- $C_{0}=\left\{\left(g_{m}\right)_{m \in \llbracket 0, M-1 \rrbracket} /\right.$ for $M$ large

$$
\begin{aligned}
& \frac{\sqrt[3]{\sum_{m=0}^{M-1} E\left|\sum_{n \in \mathrm{Z}} C_{m, n}^{R} g_{m, n}^{R}(t)-C_{m, n}^{I} g_{m, n}^{I}(t)\right|^{3}}}{\sqrt{\frac{\sigma_{c}^{2}}{2} \sum_{m=0}^{M-1} \sum_{n \in \mathrm{Z}}\left|g_{m}(t-n T)\right|^{2}}}<\epsilon_{M} \ll 1, \\
& \left.\frac{\sqrt[3]{\sum_{m=0}^{M-1} E\left|\sum_{n \in \mathrm{Z}} C_{m, n}^{R} g_{m, n}^{I}(t)+C_{m, n}^{I} g_{m, n}^{R}(t)\right|^{3}}}{\sqrt{\frac{\sigma_{c}^{2}}{2} \sum_{m=0}^{M-1} \sum_{n \in \mathrm{Z}}\left|g_{m}(t-n T)\right|^{2}}}<\epsilon_{M}^{\prime} \ll 1\right\}
\end{aligned}
$$

$C_{0}$ is the condition that we need to apply CLT. We considered Assumption 2 because the expression of $C_{0}$ is not intuitive.

- $C_{1}=\left\{\left(g_{m}\right)_{m \in \llbracket 0, M-1 \rrbracket} /\right.$ for large $M \max _{m, t}$ $\sum_{n \in Z}\left|g_{m}(t-n T)\right|=B_{1}<+\infty$ and $\min _{m, t} \sum_{n \in Z}$ $\left.\left|g_{m}(t-n T)\right|^{2}=A_{2}>0\right\} . C_{1}$ is the set of functions that satisfy Assumption 2. $C_{1}$ is stronger than $C_{0}$.

- $C_{2}=\left\{\left(g_{m}\right)_{m \in \llbracket 0, M-1 \rrbracket} / \forall m \in \llbracket 0, M-1 \rrbracket \sum_{n \in Z}\right.$ $\left.\left|g_{m, n}(t)\right|^{2}=\sum_{n \in Z}\left|g_{0, n}(t)\right|^{2}\right\} . C_{2}$ is stronger than $C_{0}$ and $C_{1}$ but easy to manipulate.

These conditions define the choice of the functions $\left(g_{m}\right)_{m \in \llbracket 0, M-1 \rrbracket}$. Figure 7 shows the inclusion relationship between the different conditions.

Thus, the optimization problem can be expressed as follows:

Optimization problem (i)

$$
\underset{\substack{\left(g_{m}\right)_{m \in \llbracket 0, M-1 \rrbracket} \\
\text { subject to }}}{\operatorname{maximize}} \int_{0}^{T} \ln \left(\begin{array}{c}
1-\mathrm{e}^{-\frac{\sum_{m=0}^{M-1}\left\|g_{m}\right\|^{2}}{P \sum_{n \in \mathrm{Z}} \sum_{m=0}^{M-1}\left|g_{m, n}(t)\right|^{2}} \gamma} \\
\left(g_{m}\right)_{m \in \llbracket 0, M-1 \rrbracket} \in C_{i} .
\end{array}\right) d t,
$$

The present study proposes to act on the modulation system to reduce the PAPR. Looking for the optimal family of modulation functions is a new approach to deal with the PAPR problem. Its advantage is that we can use, at the same time, one of the previous PAPR reduction techniques to get an even smaller PAPR.

\section{Conclusions}

In this paper, we make several derivations to achieve the more general CDF approximation of the PAPR in the sense that transmitted symbols can be carried by any family of functions. First, we define the GWMC system and derive a general approximation of its PAPR distribution for both finite and infinite integration time. We conclude that the PAPR distribution depends on the family of functions used in the modulation, but for an infinite observation duration, large peaks cannot be avoided, even if we change the family of modulation functions. To show the importance of developed expressions, we illustrate their applications: the simple computation of the PAPR distribution of any modulation system, the characterization of the CCDF of the PAPR for different transmission and observation scenarios, as well as the modelling of the PAPR reduction problem as a constrained optimization problem.

The remaining difficulty is to solve the optimization problem. This needs to be addressed by future studies. Other constraints should be added, which take into account the required performance of the system, such as the bit error rate (BER) and the implementation complexity. The solution of this constrained optimization problem 


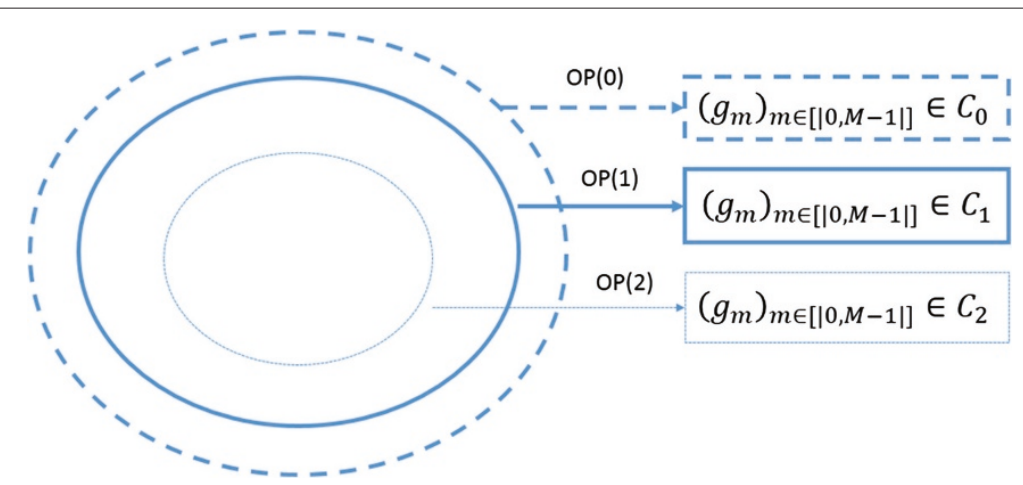

Figure 7 Relationship between the optimization problem's constraints.

can lead to a new system of multi-carrier modulation featuring an optimal performance.

\section{Appendix 1}

\section{Berry-Esseen theorem}

In probability theory, the CLT states that, under certain circumstances, the probability distribution of the scaled mean of a random sample converges to a normal distribution as the sample size increases to infinity. Under stronger assumptions, the Berry-Esseen theorem, or Berry-Esseen inequality, specifies the rate at which this convergence takes place by giving a bound on the maximal error of approximation between the normal distribution and the true distribution of the scaled sample mean. In the case of independent samples, the convergence rate is $\sqrt{\frac{1}{M}}$. In general, we have a good approximation for the Gaussian curve for $M>8$.

In our case, we perform simulations by considering the number of carriers to be equal to $M=64>8$. Under this assumption, the following Lyapunov's condition:

$$
\begin{aligned}
& \lim _{M \rightarrow+\infty} \frac{\sqrt[3]{\sum_{m=0}^{M-1} E\left|\sum_{n \in \mathrm{Z}} C_{m, n}^{R} g_{m, n}^{R}(t)-C_{m, n}^{I} g_{m, n}^{I}(t)\right|^{3}}}{\sqrt{\frac{\sigma_{c}^{2}}{2} \sum_{m=0}^{M-1} \sum_{n \in \mathrm{Z}}\left|g_{m}(t-n T)\right|^{2}}}=0 \\
& \text { and } \lim _{M \rightarrow+\infty} \frac{\sqrt[3]{\sum_{m=0}^{M-1} E\left|\sum_{n \in \mathrm{Z}} C_{m, n}^{R} g_{m, n}^{I}(t)+C_{m, n}^{I} g_{m, n}^{R}(t)\right|^{3}}}{\sqrt{\frac{\sigma_{c}^{2}}{2} \sum_{m=0}^{M-1} \sum_{n \in \mathrm{Z}}\left|g_{m}(t-n T)\right|^{2}}}=0
\end{aligned}
$$

becomes

$$
\begin{gathered}
\frac{\sqrt[3]{\sum_{m=0}^{M-1} E\left|\sum_{n \in \mathrm{Z}} C_{m, n}^{R} g_{m, n}^{R}(t)-C_{m, n}^{I} g_{m, n}^{I}(t)\right|^{3}}}{\sqrt{\frac{\sigma_{c}^{2}}{2} \sum_{m=0}^{M-1} \sum_{n \in Z}\left|g_{m}(t-n T)\right|^{2}}}<\epsilon_{M} \ll 1 \\
\text { and } \frac{\sqrt[3]{\sum_{m=0}^{M-1} E\left|\sum_{n \in \mathrm{Z}} C_{m, n}^{R} g_{m, n}^{I}(t)+C_{m, n}^{I} g_{m, n}^{R}(t)\right|^{3}}}{\sqrt{\frac{\sigma_{c}^{2}}{2} \sum_{m=0}^{M-1} \sum_{n \in \mathrm{Z}}\left|g_{m}(t-n T)\right|^{2}}}<\epsilon_{M}^{\prime} \ll 1 .
\end{gathered}
$$

\section{Appendix 2}

\section{Distribution of $X^{R}(t)$}

A. Lyapunov CLT

Let us suppose that $X_{1}, X_{2}, \ldots$ is a sequence of independent random variables, each with finite expected value $\mu_{m}$ and a variance $\sigma_{m}^{2}$. Let us define

$$
s_{M}^{2}=\sum_{m=1}^{M} \sigma_{m}^{2}
$$

and the third-order centred moments

$$
r_{M}^{3}=\sum_{m=1}^{M} E\left(\left|X_{m}-\mu_{m}\right|^{3}\right) \text {. }
$$

Lyapunov's condition is

$$
\lim _{M \rightarrow+\infty} \frac{r_{M}}{s_{M}}=0
$$

Let us put $S_{M}=X_{1}+X_{2}+\cdots+X_{M}$. The expectation of $S_{M}$ is $m_{M}=\sum_{m=1}^{M} \mu_{m}$ and its standard deviation is $s_{M}$. If Lyapunov's condition is satisfied, then $\frac{S_{M}-m_{M}}{s_{M}}$ converges in distribution to a standard normal random variable, as $M$ goes to infinity:

$$
\frac{S_{M}-m_{M}}{s_{M}} \sim \mathcal{N}(0,1)
$$

\section{B. Lyapunov's condition for GWMC}

Property 1. (Lyapunov's condition)

When Assumption 2 holds, there are constants $A_{i}>0$ and $B_{2}<+\infty$ such that for all $m, t$

$$
\begin{aligned}
& 0<A_{1} \leq \sum_{n \in Z}\left|g_{m}(t-n T)\right| \leq B_{1}<+\infty \\
& \text { and } 0<A_{2} \leq \sum_{n \in Z}\left|g_{m}(t-n T)\right|^{2} \leq B_{2}<+\infty
\end{aligned}
$$

Moreover,

$\frac{\sqrt[3]{\sum_{m=0}^{M-1} E\left|\sum_{n \in Z} C_{m, n}^{R} g_{m, n}^{R}(t)-C_{m, n}^{I} g_{m, n}^{I}(t)\right|^{3}}}{\sqrt{\frac{\sigma_{c}^{2}}{2} \sum_{m=0}^{M-1} \sum_{n \in Z}\left|g_{m}(t-n T)\right|^{2}}}<\epsilon_{M} \ll 1$, 
and $\frac{\sqrt[3]{\sum_{m=0}^{M-1} E\left|\sum_{n \in Z} C_{m, n}^{R} g_{m, n}^{I}(t)+C_{m, n}^{I} g_{m, n}^{R}(t)\right|^{3}}}{\sqrt{\frac{\sigma_{c}^{2}}{2} \sum_{m=0}^{M-1} \sum_{n \in Z}\left|g_{m}(t-n T)\right|^{2}}}<\epsilon_{M}^{\prime} \ll 1$.

Proof. Let $\max _{m, t} \sum_{n \in \mathrm{Z}}\left|g_{m}(t-n T)\right|=B_{1}<+\infty$ and $\min _{m, t} \sum_{n \in \mathrm{Z}}\left|g_{m}(t-n T)\right|^{2}=A_{2}>0$. We have

$$
\begin{gathered}
\sum_{n \in Z}\left|g_{m}(t-n T)\right| \leq B_{1}<+\infty \\
\Rightarrow \quad \sum_{n \in Z}\left|g_{m}(t-n T)\right|^{2} \leq B_{1}^{2}<+\infty \\
\Rightarrow \max _{m, t} \sum_{n \in Z}\left|g_{m}(t-n T)\right|^{2}=B_{2} \leq B_{1}^{2}<+\infty,
\end{gathered}
$$

and we have

$$
\begin{aligned}
\sum_{n \in \mathrm{Z}}\left|g_{m}(t-n T)\right|^{2} & \geq A_{2}>0 \\
\Rightarrow \quad \sum_{n \in \mathrm{Z}}\left|g_{m}(t-n T)\right| & \geq \sqrt{\sum_{n \in \mathrm{Z}}\left|g_{m}(t-n T)\right|^{2}} \geq \sqrt{A_{2}} \\
\Rightarrow \min _{m, t} \sum_{n \in \mathrm{Z}}\left|g_{m}(t-n T)\right| & =A_{1} \geq \sqrt{A_{2}}>0 .
\end{aligned}
$$

We want to prove the validity of Lyapunov'condition. We have

$$
\frac{1}{\sqrt{B_{2} M \frac{\sigma_{c}^{2}}{2}}} \leq \frac{1}{\sqrt{\frac{\sigma_{c}^{2}}{2} \sum_{m=0}^{M-1} \sum_{n \in \mathrm{Z}}\left|g_{m}(t-n T)\right|^{2}}} \leq \frac{1}{\sqrt{A_{2} M \frac{\sigma_{c}^{2}}{2}}}
$$

and we have

$$
\begin{gathered}
\left|C_{m, n}^{R} g_{m, n}^{R}(t)\right| \leq \underbrace{\max _{m, n} C_{m, n}^{R}}_{C_{R}}\left|g_{m, n}^{R}(t)\right| \text { and } \\
\left|C_{m, n}^{I} g_{m, n}^{I}(t)\right| \leq \underbrace{\max _{m, n} C_{m, n}^{I}}_{C_{I}}\left|g_{m, n}^{I}(t)\right| \\
\sum_{n \in Z}\left|C_{m, n}^{R} g_{m, n}^{R}(t)\right| \leq C_{R} B_{1} \text { and } \\
\sum_{n \in Z}\left|C_{m, n}^{I} g_{m, n}^{I}(t)\right| \leq C_{I} B_{1}
\end{gathered}
$$

$$
\text { then } \begin{aligned}
& \left|\sum_{n \in \mathrm{Z}} C_{m, n}^{R} g_{m, n}^{R}(t)-C_{m, n}^{I} g_{m, n}^{I}(t)\right| \\
\leq & \left|\sum_{n \in \mathrm{Z}} C_{m, n}^{R} g_{m, n}^{R}(t)\right|-\left|\sum_{n \in \mathrm{Z}} C_{m, n}^{I} g_{m, n}^{I}(t)\right| \\
\leq & \sum_{n \in \mathrm{Z}}\left|C_{m, n}^{R} g_{m, n}^{R}(t)\right|-\sum_{n \in \mathrm{Z}}\left|C_{m, n}^{I} g_{m, n}^{I}(t)\right| \\
\leq & C_{R} B_{1}+C_{I} B_{1}
\end{aligned}
$$

and so

$$
\begin{aligned}
\left|\sum_{n \in \mathrm{Z}} C_{m, n}^{R} g_{m, n}^{R}(t)-C_{m, n}^{I} g_{m, n}^{I}(t)\right|^{3} & \leq\left(C_{R} B_{1}+C_{I} B_{1}\right)^{3} \\
\text { thus } E\left(\left|\sum_{n \in \mathrm{Z}} C_{m, n}^{R} g_{m, n}^{R}(t)-C_{m, n}^{I} g_{m, n}^{I}(t)\right|^{3}\right) & \leq\left(C_{R} B_{1}+C_{I} B_{1}\right)^{3} .
\end{aligned}
$$

From Equations 44 and 45, we have

$$
\begin{aligned}
& \frac{\sqrt[3]{\sum_{m=0}^{M-1} E\left|\sum_{n \in \mathrm{Z}} C_{m, n}^{R} g_{m, n}^{R}(t)-C_{m, n}^{I} g_{m, n}^{I}(t)\right|^{3}}}{\sqrt{\frac{\sigma_{c}^{2}}{2} \sum_{m=0}^{M-1} \sum_{n \in \mathrm{Z}}\left|g_{m}(t-n T)\right|^{2}}} \\
& \leq \frac{\sqrt[3]{M\left(C_{R} B_{1}+C_{I} B_{1}\right)^{3}}}{\sqrt{A_{2} M \frac{\sigma_{c}^{2}}{2}}} .
\end{aligned}
$$

For large $M$, we have

$$
\begin{gathered}
\frac{\sqrt[3]{\sum_{m=0}^{M-1} E\left|\sum_{n \in \mathrm{Z}} C_{m, n}^{R} g_{m, n}^{R}(t)-C_{m, n}^{I} g_{m, n}^{I}(t)\right|^{3}}}{\sqrt{\frac{\sigma_{c}^{2}}{2} \sum_{m=0}^{M-1} \sum_{n \in \mathrm{Z}}\left|g_{m}(t-n T)\right|^{2}}} \\
<\epsilon_{M}=O\left(M^{-1 / 6}\right) \ll 1 .
\end{gathered}
$$

We can prove the second part of Lyapunov's condition by proceeding similarly. Assumption 2 is then a sufficient condition of Lyapunov's condition.

\section{Application of Lyapunov CLT}

Let us put $X_{m}^{R}(t)=\sum_{n \in \mathrm{Z}} C_{m, n}^{R} g_{m, n}^{R}(t)-C_{m, n}^{I} g_{m, n}^{I}(t)$ such that $X^{R}(t)=\sum_{m=0}^{M-1} X_{m}^{R}(t)$. In our case, we have $X_{0}^{R}(t)$, $X_{1}^{R}(t), X_{2}^{R}(t), \ldots, X_{M-1}^{R}(t)$ is a sequence of independent random variables (Assumption 1 ). And we have

- From Equation $4, \forall m \in \llbracket 0, M-1 \rrbracket E\left(X_{m}^{R}(t)\right)=0$.

- $\operatorname{Var}\left(X_{m}^{R}(t)\right)=\sum_{n \in \mathrm{Z}} \frac{\sigma_{c}^{2}}{2}\left|g_{m, n}(t)\right|^{2}$. 
In fact,

$$
\begin{aligned}
\operatorname{Var}\left(X_{m}^{R}(t)\right)= & E\left(X_{m}^{R}(t)^{2}\right) \\
= & E\left(\sum_{n, p \in \mathrm{Z}}\left[C_{m, n}^{R} g_{m, n}^{R}(t)-C_{m, n}^{I} g_{m, n}^{I}(t)\right]\right. \\
& \left.\times\left[C_{m, p}^{R} g_{m, p}^{R}(t)-C_{m, p}^{I} g_{m, p}^{I}(t)\right]\right) \\
= & E\left(\sum _ { n , p \in \mathrm { Z } } \left[C_{m, n}^{R} C_{m, p}^{R} g_{m, n}^{R}(t) g_{m, p}^{R}(t)\right.\right. \\
& -C_{m, n}^{R} C_{m, p}^{I} g_{m, n}^{R}(t) g_{m, p}^{I}(t) \\
& -C_{m, n}^{I} C_{m, p}^{R} g_{m, n}^{I}(t) g_{m, p}^{R}(t) \\
& \left.\left.+C_{m, n}^{I} C_{m, p}^{I} g_{m, n}^{I}(t) g_{m, p}^{I}(t)\right]\right) \\
= & \sum_{n \in \mathrm{Z}} \frac{\sigma_{c}^{2}}{2}\left[\left(g_{m, n}^{R}(t)\right)^{2}+\left(g_{m, n}^{I}(t)\right)^{2}\right] \quad(\mathrm{Eq} . \\
= & \sum_{n \in \mathrm{Z}} \frac{\sigma_{c}^{2}}{2}\left|g_{m, n}(t)\right|^{2} .
\end{aligned}
$$$$
=\sum_{n \in Z} \frac{\sigma_{c}^{2}}{2}\left[\left(g_{m, n}^{R}(t)\right)^{2}+\left(g_{m, n}^{I}(t)\right)^{2}\right] \quad(\text { Eq. 2, 3, 4, 5) }
$$

- $r_{M}=\sqrt[3]{\sum_{m=0}^{M-1} E\left|\sum_{n \in \mathrm{Z}} C_{m, n}^{R} g_{m, n}^{R}(t)-C_{m, n}^{I} g_{m, n}^{I}(t)\right|^{3}}$.

- $s_{M}=\sqrt{\sum_{m=0}^{M-1} \operatorname{Var}\left(X_{m}^{R}\right)}=\sqrt{\frac{\sigma_{c}^{2}}{2} \sum_{m=0}^{M-1} \sum_{n \in \mathrm{Z}}\left|g_{m, n}(t)\right|^{2}}$.

From Assumption 2, we have $\lim _{M \rightarrow+\infty} \frac{r_{M}}{s_{M}}=0$. Lyapunov's condition is then satisfied. By means of Lyapunov's CLT, we get for large $M$

$$
\begin{aligned}
\frac{\sum_{m=0}^{M-1} X_{m}^{R}(t)}{\frac{\sigma_{c}^{2}}{2} \sum_{n \in \mathrm{Z}} \sum_{m=0}^{M-1}\left|g_{m, n}(t)\right|^{2}} & \sim \mathcal{N}(0,1) \\
\sum_{m=0}^{M-1} X_{m}^{R}(t) & \sim \mathcal{N}\left(0, \frac{\sigma_{c}^{2}}{2} \sum_{n \in \mathrm{Z}} \sum_{m=0}^{M-1}\left|g_{m, n}(t)\right|^{2}\right) .
\end{aligned}
$$

\section{Appendix 3}

\section{Average power's expression}

The average power is calculated as follows:

$$
\begin{aligned}
P_{c, \text { mean }}= & \lim _{t_{0} \rightarrow+\infty} \frac{1}{2 t_{0}} \int_{-t_{0}}^{t_{0}} E\left(|X(t)|^{2}\right) d t \\
= & \lim _{t_{0} \rightarrow+\infty} \frac{1}{2 t_{0}} \int_{-t_{0}}^{t_{0}} \\
& E\left(\sum_{m, m^{\prime}=0}^{M-1} \sum_{n, n^{\prime} \in Z} C_{m, n} C_{m^{\prime}, n^{\prime}}^{*} g_{m, n}(t) g_{m^{\prime}, n^{\prime}}^{*}(t)\right) d t \\
\text { Assumption } 1 & \lim _{t_{0} \rightarrow+\infty} \frac{1}{2 t_{0}} \int_{-t_{0}}^{t_{0}} \sum_{m=0}^{M-1} \sum_{n \in Z} \sigma_{c}^{2}\left|g_{m, n}(t)\right|^{2} d t \\
= & \sigma_{c}^{2} \lim _{t_{0} \rightarrow+\infty} \frac{1}{2 t_{0}} \sum_{m=0}^{M-1} \int_{-t_{0}}^{t_{0}} \sum_{n \in Z}\left|g_{m, n}(t)\right|^{2} d t .
\end{aligned}
$$

Let us put $t_{0}=\frac{K T}{2}, K \in \mathrm{N}$

$$
P_{c \text {,mean }}=\frac{\sigma_{c}^{2}}{T} \lim _{K \rightarrow+\infty} \frac{1}{K} \sum_{m=0}^{M-1} \int_{-\frac{K T}{2}}^{\frac{K T}{2}} \sum_{n \in Z}\left|g_{m, n}(t)\right|^{2} d t .
$$

We notice that $t \mapsto \sum_{n \in Z} \sum_{m=0}^{M-1}\left|g_{m}(t-n T)\right|^{2}$ is periodic with a period $T$, so

$$
\begin{aligned}
P_{c \text {,mean }} & =\frac{\sigma_{c}^{2}}{T} \sum_{m=0}^{M-1} \int_{-\frac{T}{2}}^{\frac{T}{2}} \sum_{n \in \mathrm{Z}}\left|g_{m}(t-n T)\right|^{2} d t \\
\stackrel{\text { (by periodicity) }}{=} & \frac{\sigma_{c}^{2}}{T} \sum_{m=0}^{M-1} \sum_{n \in \mathrm{Z}} \int_{n T-\frac{T}{2}}^{n T+\frac{T}{2}}\left|g_{m}(t-n T)\right|^{2} d t \\
& =\frac{\sigma_{c}^{2}}{T} \sum_{m=0}^{M-1} \int_{-\infty}^{+\infty}\left|g_{m}(t)\right|^{2} d t \\
P_{c, \text { mean }} & =\frac{\sigma_{c}^{2}}{T} \sum_{m=0}^{M-1}\left\|g_{m}\right\|^{2} .
\end{aligned}
$$

\section{Appendix 4}

Upper bound of the PAPR [29]

Let $M$ be a finite number of carriers and $\left(g_{m}\right)_{m \in \llbracket 0, M-1 \rrbracket}$ a family of modulation functions that satisfies $A_{i}=\min _{m, t} \sum_{n \in Z}\left|g_{m}(t-n T)\right|^{i}>0 \quad$ and $\quad B_{i}=\max _{m, t}$ $\sum_{n \in Z}\left|g_{m}(t-n T)\right|^{i}<+\infty$ for $i \in\{1,2\}$. For any observation duration $I$ and any input symbols, we have

$$
\operatorname{PAPR}_{c}(I)=\frac{\max _{t \in I}|X(t)|^{2}}{P_{c \text {, mean }}} .
$$

We have

$$
\begin{aligned}
\max _{t \in I}|X(t)| & =\max _{t \in I}\left|\sum_{n \in Z} \sum_{m=0}^{M-1} C_{m, n} g_{m}(t-n T)\right| \\
& \leq \max _{t \in I} \sum_{m=0}^{M-1}\left|\sum_{n \in Z} C_{m, n} g_{m}(t-n T)\right| \\
& \leq \max _{m, n} \sum_{m=0}^{M-1}\left|C_{m, n}\right| \sum_{n \in Z}\left|g_{m}(t-n T)\right| \\
& \leq M \max _{m, n}\left|C_{m, n}\right| B_{1},
\end{aligned}
$$

and we have

$$
\begin{aligned}
& P_{c, \text { mean }}=\frac{\sigma_{c}^{2}}{T} \sum_{m=0}^{M-1} \int_{-\frac{T}{2}}^{\frac{T}{2}} \sum_{n \in \mathrm{Z}}\left|g_{m}(t-n T)\right|^{2} d t, \\
& \text { hence } M A_{2} \sigma_{C}^{2} \leq P_{c \text {, mean }} \leq M B_{2} \sigma_{C}^{2} .
\end{aligned}
$$

Thus,

$$
\operatorname{PAPR}_{c}(I) \leq \frac{\max _{m, n}\left|C_{m, n}\right|^{2} B_{1}^{2}}{\sigma_{C}^{2} A_{2}} M .
$$




\section{Abbreviations}

ADSL: asymmetric digital subscriber line; BER: bit error rate; BFDM: biorthogonal frequency division multiplexing; CDF: cumulative distribution function; CCDF: complementary cumulative distribution function; CLT: central limit theorem; DAB: Digital Audio Broadcasting; DCT: discrete cosine transform; DVB-H: Digital Video Broadcasting-Handheld; DVB-T: Digital Video Broadcasting-Terrestrial; EGF: extended Gaussien function; FSK: frequency Shift Keying; GWMC: generalized waveforms for multi-carrier; GCLT: generalized central limit theorem; HiperLAN/2: high performance radio LAN; HPA: highpower amplifier; IOTA: isotropic orthogonal transform algorithm; LTE: Long Term Evolution; NOFDM: non-orthogonal frequency division multiplexing; OFDM: orthogonal frequency division multiplexing; OQAM: offset quadrature amplitude modulation; PAPR: peak-to-average power ratio; PSK: phase shift keying; QAM: quadrature amplitude modulation; QPSK: quadrature phase shift keying; SRRC: square root raised cosine; WH-HT: Walsh-Hadamard multi-carrier.

\section{Competing interests}

The authors declare that they have no competing interests.

\section{Acknowledgements}

This work has received a French state support granted to the CominLabs excellence laboratory and managed by the National Research Agency in the 'Investing for the Future' program under reference no. ANR-10-LABX-07-01. The authors would also like to thank the 'Région Bretagne', France for supporting this work.

\section{Author details}

${ }^{1}$ SUPELEC/IETR, Cesson-Sévigné Cedex 35576, France. ${ }^{2}$ Inria, Bretagne Atlantique, Campus de Beaulieu, Rennes Cedex 35042, France.

Received: 1 December 2013 Accepted: 17 July 2014

Published: 30 July 2014

\section{References}

1. RW Chang, Synthesis of band-limited orthogonal signals for multichannel data transmission. Bell Sys. Tech. J. 45, 1775-1796 (1966)

2. SWeinstein, P Ebert, Data transmission by frequency-division multiplexing using the discrete Fourier transform. Commun. Technol. IEEE Trans. 19(5), 628-634 (1971)

3. R Lassalle, M Alard, Principles of modulation and channel coding for digital broadcasting for mobile receivers. EBU Tech. Rev. 224, 168-190 (1987)

4. ETSI TS 101 388 V1. 3.1 (2002-05), Asymmetric Digital Subscriber Line (ADSL)-European specific requirements (2002)

5. Digital Audio Broadcasting (DAB); DAB to mobile, portable and fixed Receivers, Tech. Rep. ETSI ETS 300401 (1995)

6. ETSI EN 302755 v1. 2.1 (2010-10) Digital Video Broadcasting (DVB), Frame structure channel coding and modulation for a second generation digital terrestrial television broadcasting system (DVB-T2)

7. ETSI TS 101475 V1. 3.1 (2001-12), Broadband radio access networks (BRAN); HIPERLAN type 2; physical (PHY) layer (2001)

8. R Van Nee, R Prasad, OFDM for Wireless Multimedia Communications. (Artech House Inc., Norwood, 2000)

9. M Hoch, S Heinrichs, JB Huber, Peak-to-average power ratio and its reduction in wavelet-OFDM, in International OFDM Workshop (Hamburg, Germany, 2011), pp. 56-60

10. M Gautier, C Lereau, M Arndt, I Lienard, PAPR analysis in wavelet packet modulation, in 3rd International Symposium On Communications, Control and Signal Processing, 2008. ISCCSP 2008 (IEEE St Julians, 2008), pp. 799-803

11. L Patidar, A Parikh, BER Comparison of DCT-based OFDM and FFT-based OFDM using BPSK modulation over AWGN and multipath Rayleigh fading channel. Int. J. Comput. Appl. 31(10), 38-41 (2011). New York, USA

12. P Chevillat, G UngerBoeck, Optimum FIR transmitter and receiver filters for data transmission over band-limited channels. Commun. IEEE Trans. 30(8), 1909-1915 (1982)

13. B Le Floch, M Alard, C Berrou, Coded orthogonal frequency division multiplex. Proc. IEEE. 83(6), 24-182 (1995)

14. A Viholainen, M Bellanger, M Huchard, Prototype filter and structure optimization. PHYDYAS project, January 2009

15. H Zhang, D Yuan, M Pätzold, Novel study on PAPRs reduction in wavelet-based multicarrier modulation systems. Digital Signal Process. 17(1), 272-279 (2007)
16. W Kozek, AF Molisch, Nonorthogonal pulseshapes for multicarrier communications in doubly dispersive channels. Selected Areas Commun. IEEE J. 16(8), 1579-1589 (1998)

17. H Bogucka, AM Wyglinski, S Pagadarai, A Kliks, Spectrally agile multicarrier waveforms for opportunistic wireless access. Commun. Mag. IEEE. 49(6), 108-115 (2011)

18. A Aboltins, Comparison of orthogonal transforms for OFDM communication system. Electronics and Electrical Engineering. 111(5), 77-80 (2011)

19. M Chafii, J Palicot, R Gribonval, Closed-Form Approximations of the PAPR Distribution for Multi-Carrier Modulation Systems. (Lisbon, Eusipco, 2014)

20. B Knaeble, Variations on the projective central limit theorem. PhD thesis, University of Utah, 2010

21. C Siclet, Application de la théorie des bancs de filtres à l'analyse et à la conception de modulations multiporteuses orthogonales et biorthogonales. PhD thesis, Rennes 1 University, France, 2002

22. A Skrzypczak, Contribution à l'étude des modulations multiporteuses OFDM/OQAM et OFDM suréchantillonnées. PhD thesis, Rennes 1 University, France, 2007

23. BG Evans, G Bi, Hardware structure for Walsh-Hadamard transforms. Electron. Lett. 34(21), 2005-2006 (1998)

24. L Wang, J Liu, PAPR reduction of OFDM signals by PTS with grouping and recursive phase weighting methods. Broadcast. IEEE Trans. 57(2), 299-306 (2011)

25. C-P Li, S-H Wang, C-L Wang, Novel low-complexity SLM schemes for PAPR reduction in ofdm systems. Signal Process. IEEE Trans. 58(5), 2916-2921 (2010)

26. D Guel, J Palicot, Transformation des techniques "ajout de signal" en techniques "tone reservation" pour la réduction du PAPR des signaux OFDM. (GRETSI, Dijon, 2009)

27. Rishabh K, K Nachiket, N Piyush. EE678 Wavelet Application Assignment, Wavelet OFDM

28. MHM Nerma, NS Kamel, V Jeoti, NA Baykara, NE Mastorakis, BER performance analysis of OFDM system based on dual-tree complex wavelet transform in AWGN channel. (Istanbul, Turkey, 2009)

29. M Chafii, J Palicot, R Gribonval, A PAPR upper bound of Generalized Waveforms for Multi-Carrier modulation systems, in ISCCSP, 6th International Symposium on Communications, Control and Signal Processing (Athens, 2014)

doi:10.1186/1687-6180-2014-121

Cite this article as: Chafii et al:: Closed-form approximations of the peakto-average power ratio distribution for multi-carrier modulation and their applications. EURASIP Journal on Advances in Signal Processing 2014 2014:121.

\section{Submit your manuscript to a SpringerOpen ${ }^{\circ}$ journal and benefit from:}

- Convenient online submission

Rigorous peer review

- Immediate publication on acceptance

- Open access: articles freely available online

- High visibility within the field

- Retaining the copyright to your article

Submit your next manuscript at $\boldsymbol{\nabla}$ springeropen.com 